\title{
Design and Evaluation of a Device for Measuring 3D-Micromotions of Press-Fit Femoral Stem Prostheses
}

\author{
Bühler D.W., Brunner P., Nolte L.-P. \\ M.E. Müller Institute for Biomechanics, University of Bern, 3010 Bern, Switzerland
}

\section{INTRODUCTION}

Primary stability of uncemented total hip replacements is regarded as a major factor necessary for good quality bony ongrowth to the prosthetic femoral stem and therefore for a successful long-term outcome [1,2]. The remodelling of the endothelial bone in contact with the implant is affected by the relative micromotions of the bone-implant interface and the stress state in the femoral bone. As a result, for optimal biological fixation, translational movements (i.e. primary stability) are of greatest interest. These interface-micromotions generally occur in all three spatial directions. Under physiologic loading, they consist of a cyclic amplitude and change in the mean, which in the cranio-caudal direction represents subsidence of the prosthesis.

Existing measurement strategies which are based on dial gauges, extensometers, LVDT's, hall effect transducers or strain gauge techniques provide information about only one component of the general three-dimensional micromovement $[3,4,5]$. Furthermore, fixation of sensors away from the point of interface motion measurement, as seen in various studies [4,5], may result in the registration of motions due to elastic deformation of the femoral or prosthetic shaft in addition to the interface motion.

The objective of this study was to develop, validate, and apply a new technique which allows the precise measurement of the isolated $3 D$ interface motion at three different points along the femoral shaft.

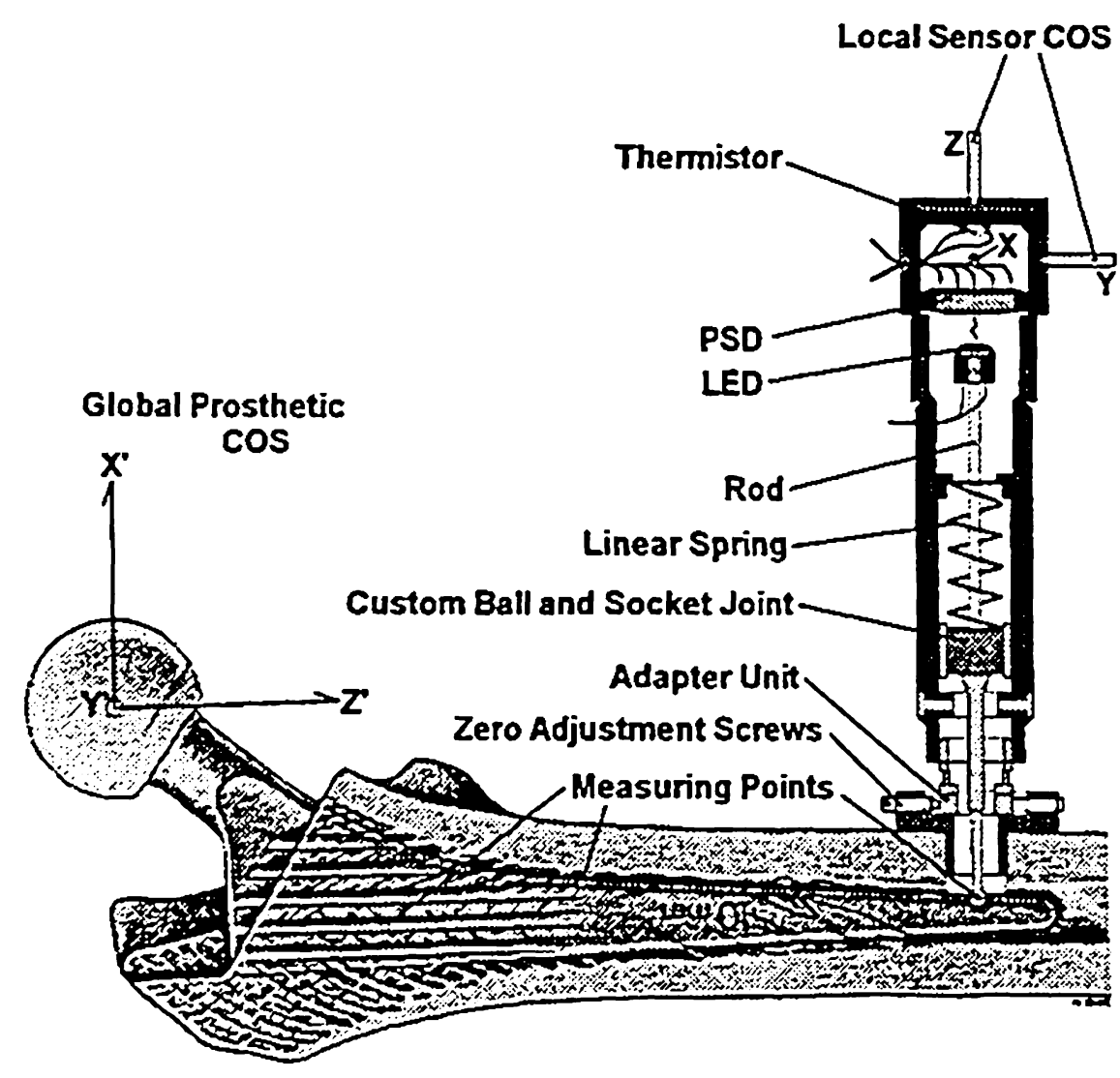

Figure 1: Conceptual Design of the Sensor

\section{MATERIALS AND METHODS}

The present measuring technique is based on a sensor combining optoelectronic with precision mechanical components. Spherical measuring tips of three sensors were placed on the prosthesis at predefined locations: the distal tip of the prosthesis and at the middle and proximal third of the femoral stem. The sensors were mounted on an adapter unit which was anchored in a transverse $9 \mathrm{~mm}$ hole drilled in the cortex. Precision, play-free, ball bearing mechanisms allowed transition of the detected motion to a infrared emitting diode on the opposite end of the sensor (Fig. 1). Photons emitted by this LED were registered by a two dimensional silicon position sensitive detector (PSD). The $X Y$-movements of the LED were measured directly by the PSD. Micromovement of the prosthesis in the $Z$-axis of the transducer brings the LED closer or farther to the photodiode, thereby increasing or decreasing the intensity of the light beam on the PSD. Since the two-dimensional position sensitive detector generates photocurrents, which sum is proportional to the intensity, the highly non-linear correlation between LEDPSD distance and light intensity was used to acquire the third dimension at the same time as measuring the $X Y$ motions. Due to the thermal response of the PSD, a ntcresistor was also integrated to exclude thermodrifts during long-term testing.

Static and dynamic validation indicated that the maximum system error was \pm 4.9 microns within a measuring range of $\pm 0.75 \mathrm{~mm}$ in each spatial orientation .

To demonstrate the utility of the sensors in interface motion measurement, an in-vitro experiment was performed. Seven paired fresh cadaveric femora were used for testing two different types of uncemented femoral stems: CLS stem (Spotorno) and Cone prosthesis (Wagner) both from Protek AG, Switzerland. Using a custom loading rig the femora were subjected to sinusoidal cyclic loading in a bi-axial materials testing machine. A cranio-caudal force Fcc with a frequency of 1 $\mathrm{Hz}$ was combined with a $0.5 \mathrm{~Hz}$ antero-posterior force Fap having a magnitude of $10 \%$ of Fcc. Loading steps of 1-, 2- and 3-times body weight (BW) were applied. Custom software allowed for real-time graphical display of the applied forces and the detected movements.

\section{RESULTS}

The postprocessing software allowed the analysis of: $(A)$ Three-dimensional motion displayed in cylindrical coordinates (Fig. 2); (B) Motion amplitude (MA), which is the difference of the measured minimum and maximum 
value for one loading cycle and (C) Total motion (TM), which is the time-based average micromotion of one loading cycle (Fig. 3).

(A) Three-Dimensional Motion: A typical 3D interface motion behavior for one loading cycle (loading step 3: Fcc $=2.07 \mathrm{kN}$; Fap=207 N) is illustrated for both types of prostheses in Fig. 2. The midshaft of both prostheses was the point with the least motion in all directions. At the distal tip the Cone prosthesis also showed very little movement. A comparably large MA was measured at the proximal position for both prostheses. This motion loop was mainly oriented in the $X ' Z$ '-plane (=frontal, see figure 1).

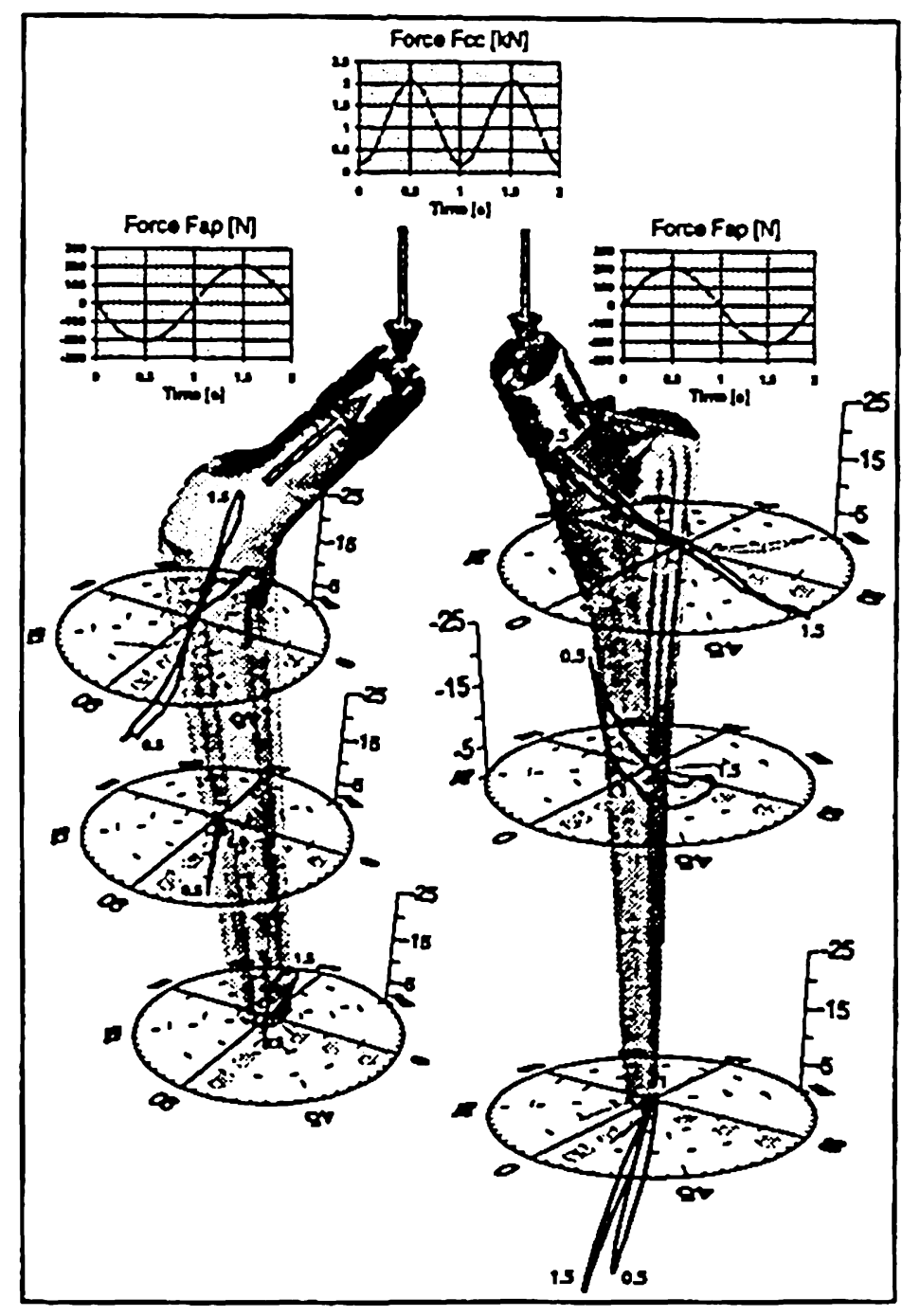

Figure 2: 3D-Motion Analysis

(B) Motion Amplitude: At higher forces the MA measured in a loading cycle increased for both prostheses. All MA values for the CLS prosthesis measured at the middle third and proximal third of the femoral stem were larger in every direction than the values for the Cone prosthesis (values for $3 \times B W$ : CLS from 20 to $87 \mu \mathrm{m}$, Cone from 12 to $35 \mu \mathrm{m})$. However, at the proximal end of the shaft the Cone prostheses allowed a larger MA (CLS from 26 to $78 \mu \mathrm{m}$, Cone from 50 to $120 \mu \mathrm{m}$ ). In most cases the largest single component of the MA was found in the mediolateral direction.

(C) Total Motion: At the end of the first and second loading step both prostheses showed relatively small TM in any of the motion directions at the various measurement points. In the third loading step TM for both prostheses became much larger with the main increase during the first 250 cycles. At the end of stage 3 the TM of the Cone was larger than the TM of the CLS prosthesis at all measuring positions. The caudal subsidence was the largest contributor to the TM for both prostheses. In $X^{\prime}$ (M-L) and ' Y' (A-P) directions the stems appeared more stable. The average TM at the distal tip of the prosthesis for all seven paired tested femora is presented in Fig. 3 (for axes orientation refer to Fig. 1).

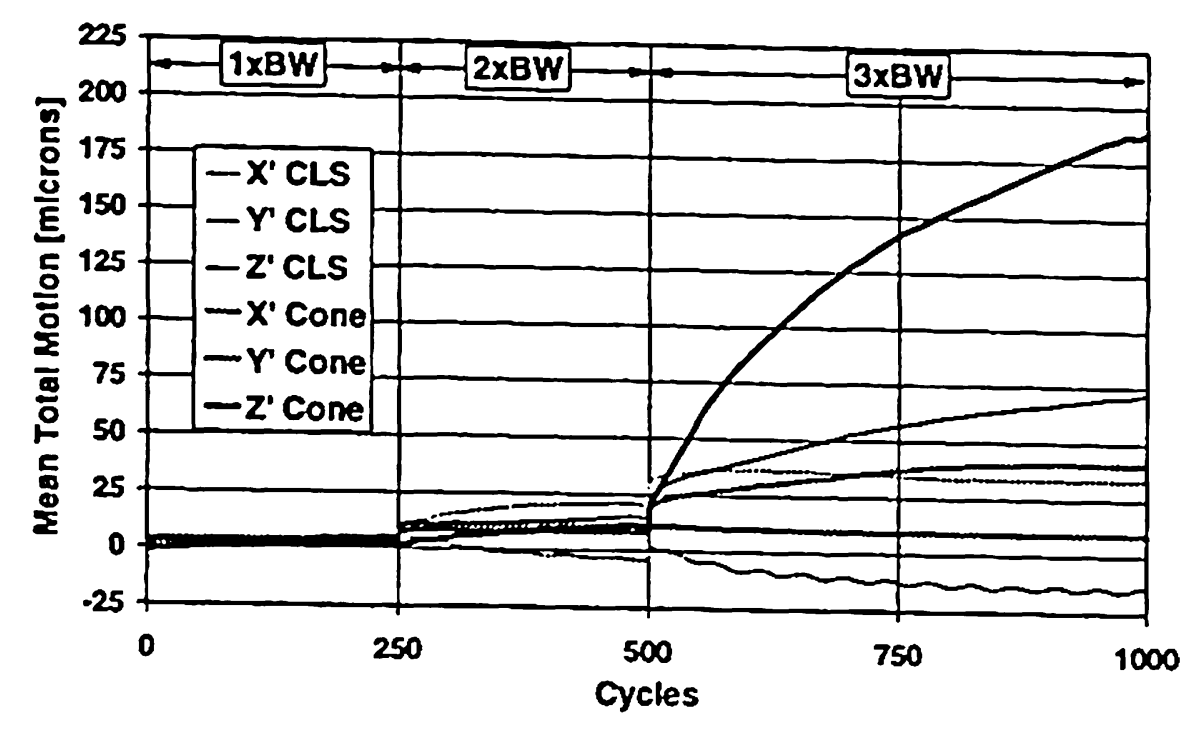

Figure 3: Mean Total Motion

\section{DISCUSSION}

The developed method seems sensitive in reflecting the actual postoperative situation of the implant. Our novel technique provided accurate and repeatable data illustrating the $3 \mathrm{D}$ interface motion of uncemented femoral stems. Furthermore, it is necessary to measure the stability of the prosthesis at several points in all three directions of the coordinate system to account for the 3D motion behavior of the implant with respect to the surrounding bone.

The two prostheses investigated in this study showed considerable differences in both their motion amplitude and total motion behavior. This reflects differences in their anchoring design. Analysis of different concepts of femoral stem fixation with the presented standardized approach may further elucidate the role of primary stability in successful long-term clinical outcomes.

In addition the investigation of biomechanical problems often makes it necessary to measure relative small translations or micromotions in the three-dimensional space. By changing the design of the adapter this easy-tohandle optical measuring system could also be used in a variety of other tasks like detection of translations of bone fragments after a fracture or the measurement of relative movements between adjacent spinal vertebrae under different loads.

\section{REFERENCES}

[1] R.M. Pilliar et al.; Clin. Orthop. 208, 108-113 (1986)

[2] R.J. Haddad Jr. et al.; J. Bone Joint Surg. 69A, 14591466 (1987)

[3] L.A. Whiteside et al.; J. Arthroplasty 8, 147-155 (1993)

[4] D.W. Burke et al.; J. Bone Joint Surg. 73B, 33-37 (1991)

[5] P.S. Walker et al.; J. Biomech. 20(7), 693-702 (1987)

\section{ACKNOWLEDGEMENTS}

This study was supported in part by Protek AG, Münsingen, Switzerland 